Article

\title{
A Gene Signature for Selecting Benefit from Hypoxia Modification of Radiotherapy for High Risk Bladder Cancer Patients
}

Lingjian Yang ${ }^{1}$, Janet Taylor ${ }^{1,2,7}$, Amanda Eustace ${ }^{1}$, Joely J. Irlam ${ }^{1}$, Helen Denley ${ }^{3}$, Peter J. Hoskin ${ }^{4}$, Jan Alsner ${ }^{5}$, Francesca M. Buffa ${ }^{6}$, Adrian L. Harris ${ }^{6}$, Ananya Choudhury ${ }^{1}$, Catharine M. L. West ${ }^{1}$

Author affiliations: ${ }^{1}$ Translational Radiobiology Group, Institute of Cancer Sciences, University of Manchester, Manchester Academic Health Science Centre, Christie Hospital, Manchester, M20 4BX, UK. ${ }^{2}$ Applied Computational Biology \& Bioinformatics Group, Paterson Institute for Cancer Research, Manchester, M20 4BX, UK. ${ }^{3}$ Department of Cellular Pathology, Manchester Royal Infirmary, Manchester, M13 9WL, UK. ${ }^{4}$ Cancer Centre, Mount Vernon Hospital, Rickmansworth Road, Northwood, Middlesex, HA6 2RN, UK. ${ }^{5}$ Department of Clinical Medicine, Aarhus University, Nørrebrogade 44, 8000 Aarhus C, Denmark. ${ }^{6}$ Weatherall Institute of Molecular Medicine, University of Oxford, Oxford, OX3 9DS, UK. ${ }^{7}$ HMDS, Leeds Cancer Centre, St James University Hospital, Leeds LS9 7TF, UK.

Running title: Bladder cancer hypoxia signature

Keywords: Bladder cancer, tumor hypoxia, gene signature, radiotherapy, hypoxia modification. 
Acknowledgements: We thank the Cancer Research UK Manchester Institute Cancer Research Molecular Biology Core Facility and the University of Manchester Clinical Immune and Molecular Monitoring Laboratory for use of Good Clinical Practice facilities. JT was funded by Cancer Research UK (grant C480/A12328). AE was funded by the Medical Research Council of the UK (grant G0801525). The work was also supported by Cancer Research UK (grant C1094/A11365) and Experimental Cancer Medicine Centre funding. FMB and ALH were funded by the Cancer Research UK, EU Metoxia, and the NIHR Biomedical Research Centre, Oxford.

Corresponding author: Prof Catharine ML West, Translational Radiobiology Group, University of Manchester, Manchester Academic Health Centre, Christie Hospital, Wilmslow Road, Manchester, M20 4BX, UK. Phone: +44 (0)161-446-3282; fax: +44 (0)161-446-8111; email: catharine.west@manchester.ac.uk

CONFLICT OF INTEREST: The authors declare no potential conflict of interest.

Word counts: 3473

Number of figures and tables: 6 


\section{TRANSLATIONAL RELEVANCE}

Hypoxia is a common component in micro-environment of most solid tumors, including bladder cancers. Adding concurrent hypoxia modification into radiotherapy improves the survival of patients with good evidence that more hypoxic tumors benefit the most from hypoxia-modifying therapy. However, there are no clinically validated biomarkers that can select the muscle invasive tumors which would benefit from adding hypoxia-modifying therapy into radical radiotherapy. A signature was derived from public databases and independently validated in a de novo cohort from the BCON trial which randomized patients to radiotherapy alone or with carbogen and nicotinamide. The signature was both prognostic and predictive of benefit from adding concurrent hypoxic modification to radiotherapy. The signature has translational relevance as tumors from the BCON trial were FFPE samples, a routine technique for preserving tissues in hospitals. The signature can aid identification of patients likely to benefit from the addition of carbogen and nicotinamide to radiotherapy. 


\section{Abstract}

PURPOSE: Hypoxia modification improves overall survival in muscle invasive bladder cancer patients who undergo radiotherapy. There is evidence that hypoxic tumors benefit most from hypoxia modification. The study aimed to identify or derive a hypoxia gene signature that predicts benefit from hypoxia-modifying treatment in bladder cancer.

PATIENTS AND METHODS: Published hypoxia signatures were tested and a new one derived by analyzing bladder cancer transcriptomic data from public databases. Tumor samples were available from the BCON phase III randomized trial of radiotherapy alone or with carbogen and nicotinamide (CON). Gene expression data were generated for 151 tumors using Affymetrix Human 1.0 Exon ST arrays and used for independent validation.

RESULTS: A 24-gene signature was derived, which was prognostic in four out of six independent surgical cohorts ( $n=679$, meta $\mathrm{HR} 2 \cdot 32,95 \% \mathrm{Cl} 1 \cdot 73-3 \cdot 12, P<0 \cdot 0001$ ). The signature was also prognostic in BCON patients receiving radiotherapy alone ( $n=75, H R$ for local relapse free survival $2 \cdot 37,95 \% \mathrm{Cl} 1 \cdot 26-4 \cdot 47, P=0 \cdot 0076)$. The signature predicted benefit from $\operatorname{CON}(n=76, \mathrm{HR} 0 \cdot 47,95 \% \mathrm{Cl} 0 \cdot 26-0 \cdot 86, P=0 \cdot 015)$. Prognostic $(P=0 \cdot 017)$ and predictive $(P=0 \cdot 058)$ significance remained after adjusting for clinicopathological variables. A test for interaction between hypoxia status and treatment arms was significant $(P=0.0094)$.

CONCLUSION: A 24-gene hypoxia signature has strong and independent prognostic and predictive value for muscle invasive bladder cancer patients. The signature can aid identification of patients likely to benefit from the addition of carbogen and nicotinamide to radiotherapy. 


\section{INTRODUCTION}

Muscle invasive carcinoma is a high risk bladder cancer subtype (1). Surgery is often the preferred treatment choice while radical radiotherapy, with the advantage of preserving the normal bladder function, is also an option (2). Hypoxia is a common micro-environmental component in most solid tumors and is associated with a poor prognosis in multiple cancer types (3-7). Adding concurrent hypoxia modification to radiotherapy can improve treatment outcomes with good evidence that more hypoxic tumors benefit most from the hypoxiamodifying therapy $(4,6,8)$.

Gene expression signatures show promise for clinical application (7). To date, hypoxia gene signatures were successfully developed for head and neck, breast, and lung cancers, and demonstrated to be not only prognostic in multiple tumor types, but also predictive of benefit from hypoxia-modifying therapy in head and neck cancers $(4,6,7,9)$. However, current evidence suggests a need to tailor signatures for different types of tumors (3). Specifically, we (6) showed that a hypoxia gene signature developed using samples from head and neck cancer biopsies failed to predict the benefit of adding hypoxia-modifying treatment to radiotherapy for bladder cancer. The aim here, therefore, was to identify a hypoxia gene signature for muscle invasive bladder cancer patients, which predicted benefit from hypoxia-modifying therapy. In order to achieve this, published signatures were tested and a bladder-specific signature derived using an approach previously proven effective by us $(6,9)$ and others $(10)$. The BCON phase III trial which randomized bladder cancer patients to radiotherapy alone or with hypoxia modification was used as a cohort to validate the predictive performance of the signatures (11). 


\section{METHODS}

\section{BCON cohort}

The study was conducted in accordance with European GCP and approved by local research ethics committee (LREC 09/H1013/24). Written informed consent was obtained. Pretreatment formalin-fixed, paraffin-embedded (FFPE) samples were available from the prospective BCON multicenter phase III clinical trial of radiotherapy alone or with carbogen and nicotinamide (CON). Patients had T1-T4a urothelial (transitional) cell bladder carcinoma and were randomized between November 2000 and April 2006. BCON patients received 55 Gy in 20 fractions in four weeks or 64 Gy in 32 fractions in 6.5 weeks daily, five times per week. Carbogen was given five minutes before and during radiotherapy. Nicotinamide (40 to $60 \mathrm{mg} / \mathrm{kg}$ ) was given 1.5 to 2 hours before each fraction.

A power calculation determined that 150 samples were required to detect difference in hazard ratio with 0.01 significance and $80 \%$ (assuming equal size of treatment arms). Supplementary Figure S1 shows the Consort diagram for the study. Among the 152 BCON patients, 75 received $\mathrm{RT}$ and 77 received $\mathrm{RT}+$ CON. Table 1 describes the cohort demographics, which are comparable to the original clinical trial (Supplementary Table S1). One patient in the RT + CON arm was excluded from the signature validation as pathological review was consistent with prostate rather than bladder cancer. Histopathology, RNA extraction, quality control and exon array hybridization are described in Supplementary Methods. The 151 BCON patients used in this study contained nine T1 tumors and one grade 2 tumor, where T2 or greater tumors and grade 3 tumors are associated with higher risk. The high risk muscle invasive nature of the cohort represents the target patient where the 
predictive signature proposed in this work should be the most relevant in clinical application.

\section{Independent cohorts}

One bladder cancer training cohort was curated from the cancer genome atlas project (TCGA) (12). TCGA has the RNA-sequencing and clinical information for 408 fresh frozen muscle invasive samples. Another six bladder cancer cohorts (GSE5287, GSE13507, GSE31684, GSE32894, GSE19915, and GSE1827) were also collected as independent validation. Procedures for normalization of transcriptomic data are provided in Supplementary Methods.

\section{Endpoints and statistical analysis}

In the BCON study, bladder tumor control was based on cyctoscopic examination at 6 month post treatment and at 6-monthly intervals for a total of 5-years. Regional and systemic assessment with CT was carried out as clinically indicated. Local relapse free survival (LRFS) was taken as time to muscle invasive tumor recurrence in bladder, locoregional failure, or death. Patients with persistent muscle-invasive disease or with no cystoscopy post treatment had their time set to zero. Overall survival (OS), defined as death from any cause, was the main clinical endpoint for surgical cohorts if available. Otherwise, disease free survival (DFS), defined as death from cancer, was used as indicated in the original publications. Patients were censored at 5-year. The chi square test was used to compare proportions across the levels of categorical factors. The Mann-Whitney $U$ test was used to 
compare median values for continuous variables between two groups. Survival estimates were performed using the Kaplan-Meier method and differences compared using the logrank test. Hazard ratios (HR) and 95\% confidence intervals $(\mathrm{Cl})$ were obtained using Cox proportional hazard model. Hazard ratios from different cohorts were combined to produce a meta-score in a fixed effect model with generic inverse variance method. All $P$-values were two sided and statistical significance was set as 0.05.

\section{Testing the predictive ability of published gene signatures}

Seven hypoxia signatures developed for tumors of different origins with prognostic value being demonstrated in independent clinical cohorts were tested in BCON $(4,7,9,13-15)$. Five bladder cancer signatures $(1,16-19)$ were also evaluated. For each signature, a Cox model was trained with the TCGA cohort and hypoxia scores derived for BCON patients with frozen gene coefficients. BCON patients were stratified into high-hypoxia and low-hypoxia groups based on median cohort scores.

\section{A novel bladder cancer hypoxia gene signature}

To derive a bladder cancer-specific hypoxia signature, a candidate list of 611 generic hypoxia regulated genes was created from a recent literature review (3). Most of the curated genes are up-regulated under hypoxia across different tumor sites. Similarly to the multi-seed approach used in our previous studies $(7,9,20)$, we hypothesized that a candidate gene is likely to be hypoxia regulated in bladder cancer if co-expressing with multiple candidate genes. Therefore, a co-expression network was constructed with two genes connected if 
positively correlated (Spearman correlation $\geq 0.5$ ) in TCGA (12). Hypoxia signature genes were selected as being prognostic $(\operatorname{Cox} P<0.05)$ and associated with poor prognosis $(\mathrm{HR}>$ 1). Hypoxia gene signature score was computed as median of gene expressions for each tumor. Details of the method are available in Supplementary Methods. The derived signature was then frozen and tested in independent validation cohorts and BCON patients. Patients were stratified into high-hypoxia and low-hypoxia groups based on the median cohort hypoxia score. In a multivariate analysis of the BCON cohort, the hypoxic status/CON was adjusted for age of diagnosis, gender, tumor stage, necrosis, and presence of carcinoma in situ (CIS).

\section{RESULTS}

\section{Testing literature gene signatures}

Among the published signatures tested, only the Lendahl hypoxia signature and Riester bladder cancer classifier had prognostic and/or predictive significance (Table 2, Supplementary Table S3).

\section{Deriving a bladder cancer-specific hypoxia gene co-expression network}

As described in the methods, in vivo transcriptomic data were integrated with knowledge of gene functions to derive bladder cancer specific hypoxia co-expression network and signature. The derived network comprised 168 candidate hypoxia genes with 458 significant interactions (Supplementary Figure S2). The high number of interactions between the 
candidate genes indicates a good likelihood of their hypoxia relevance in bladder carcinoma. The final bladder cancer hypoxia signature derived comprised 24 genes, high expression of which was significantly associated with poor prognosis (Supplementary Table S4).

Copy number variation and methylation data were analyzed to investigate the underlying associations with the 24-gene panel. Copy number variation and methylation status was correlated with the expression of the 24 genes in our signature using data for TCGA tumor samples. The average correlation between copy number variation of the 24 genes and their corresponding gene expression across the de novo gene panel was 0.16. A null distribution was constructed by calculating the mean correlation values of 10000 random gene sets of the same size from the whole platform. The correlation for the 24-gene panel was not significantly higher than the random gene sets $(P=0.99)$, suggesting that the expression levels of the signature genes were not driven by copy number alteration. A similar analysis was performed for methylation data, where the gene panel had an average correlation of 0.36 between methylation status and gene expression for the 24 genes, which was significantly lower than that of the random gene sets $(P=0.0008)$. This analysis suggests there is a significant association between methylation status and the expression of the genes in our bladder signature.

\section{Testing the de novo hypoxia signature in independent cohorts}

The prognostic value of the frozen signature in chemotherapy or cystectomy-treated patients was validated in six publically available bladder cancer cohorts totaling 679 patients which were independent from the discovery cohort. Kaplan-Meier plots are provided in 
Figure 1. Patients stratified as high hypoxia by the de novo signature were significantly associated with poor prognosis in GSE13507, GSE31684, GSE32894, and GSE1827. Similar trends can be observed in the other two cohorts (GSE5287 and GSE19915) where the signature had borderline significance. Meta-analysis of the six cohorts revealed a HR of $2 \cdot 32$ (95\% Cl 1·73-3.12, $P<0 \cdot 0001$ ) for hypoxic tumors (Supplementary Figure S3).

\section{Testing the de novo hypoxia signature in BCON patients}

In the BCON cohort, patients stratified as high hypoxia by the signature had higher tumor stage $(P=0.03$, Supplementary Table S5). High hypoxia also corresponded to lower pretreatment hemoglobin levels $(P=0 \cdot 04$, Supplementary Table S5). Protein expression data for three well-known hypoxia biomarkers (CAIX, HIF-1 $\alpha$, and GLUT1) were available for 127, 92 and 95 of the BCON gene expression cohort, respectively (21). For each protein marker, tumors were stratified into two groups based on upper quartile, median or lower quartile. A $t$-test (two-tailed) was applied to determine if the 24-gene signature score was significantly different in high and low protein expression groups. The 24-gene signature score was significantly higher in tumors with high CAIX protein expression $(P=0.013$, upper quartile, Supplementary Figure S4A). Tumors with high HIF-1 $\alpha$ protein expression had a trend towards having significantly higher 24-gene signature score $(P=0.081$, lower quartile, Supplementary Figure S4B). No significant association was found between the signature and GLUT1 expression.

The prognostic value of the signature was examined in the BCON radiotherapy arm (Figure 2A). Patients categorized as more hypoxic had a significantly poorer LPFS than those 
categorized as less hypoxic $(P=0 \cdot 0076, \mathrm{HR} 2 \cdot 37,95 \% \mathrm{Cl} 1 \cdot 26-4 \cdot 47)$. Tumor hypoxia retained prognostic significance in multivariate analysis $(P=0 \cdot 017, \mathrm{HR} 2 \cdot 25$, Table 3$)$. The signature had no prognostic significance in patients receiving $\mathrm{RT}+\mathrm{CON}$ (Figure 2B).

The 24-gene signature also predicted benefit from adding CON to RT. Patients with highhypoxia scores receiving RT + CON had an improved LPFS rate than those undergoing RT alone $(P=0 \cdot 015, \mathrm{HR} 0 \cdot 47,95 \% \mathrm{Cl} 0 \cdot 26-0 \cdot 86$, Figure $3 \mathrm{~A})$. Adding $\mathrm{CON}$ into RT was associated with borderline significance in multivariate analysis $(P=0.058, \mathrm{HR} 0.52$, Table 3$)$. Patients with low-hypoxia scores derived no benefit in from $\operatorname{CON}(P=0 \cdot 21$, Figure 3B). Hazard ratio, $95 \%$ confidence intervals and $P$ value for prognostic testing of RT-treated patients and patients with high hypoxia signature score were detailed in Table 3. A test for interaction between hypoxia signature and treatment with the entire cohort showed that response to CON was significantly different in more hypoxic tumors than normoxic tumors, for both binary hypoxic status $(P=0.0094)$ and continuous hypoxia score $(P=0.045)$.

The potential benefit of integrating the 24-gene signature with the Riester signature (16) was also investigated. Stratification of BCON patients into four categories based on both 24gene hypoxia signature and Riester risk signature improved the prognostic value on patients receiving RT only (Supplementary Figure S5). Adding the Riester signature into the de novo hypoxia signature also led to increased predictive significance in patients with both high hypoxia and high Riester signature scores ( $n=40, P=0.0082, \mathrm{HR} 0 \cdot 32,95 \% \mathrm{Cl}$ 0.14-0.75, Supplementary Figure S6). Adding the Lendahl signature (15) into the 24-gene signature did not improve its prognostic significance (Supplementary Figure S7) but resulted in higher predictive power ( $n=43, P=0 \cdot 014$, HR 0.32, 95\% Cl 0·13-0·79, Supplementary Figure S8). 
The prognostic and predictive performance of CAIX, HIF-1 $\alpha$, GLUT1, and necrosis were assessed in the BCON gene expression cohort used in this study. For each protein marker, tumors were stratified into two groups based on upper quartile, median or lower quartile. Tumors with high CAIX expression (upper quartile split) had poorer LPFS when treated with RT alone ( $n=64, P=0 \cdot 022, \operatorname{HR} 2 \cdot 21,95 \% \mathrm{Cl} 1 \cdot 12-4 \cdot 37)$ and derived benefit from CON ( $n=32$, $P=0 \cdot 017, \mathrm{HR} 0 \cdot 32,95 \% \mathrm{Cl} 0 \cdot 13-0 \cdot 82$ ). A significant interaction between CAIX status and intervention was detected $(P=0 \cdot 019)$. High GLUT1 expression (median split) had no prognostic value in RT-treated patients $(P=0 \cdot 20)$, while there was a trend for significance in prediction of benefit of $\operatorname{CON}(n=48, P=0.077, \mathrm{HR} 0.51,95 \% \mathrm{Cl} 0 \cdot 24-1 \cdot 08)$. A test of interaction also showed borderline significance $(P=0.096)$. No prognostic or predictive significance was found for HIF-1 $\alpha$ expression in this cohort. Necrosis was associated with a poor prognosis $(n=75, P=0 \cdot 029, \operatorname{HR} 1 \cdot 97,95 \% \mathrm{Cl} 1 \cdot 08-3 \cdot 60)$, predictive value $(n=80$, $P=0 \cdot 0051, \mathrm{HR} 0 \cdot 43,95 \% \mathrm{Cl} 0 \cdot 24-0 \cdot 78)$, and a significant interaction $(P=0.002)$. The potential benefit of combining the 24-gene signature with necrosis was then investigated. CON was associated with lower HR in tumors having both high 24-gene signature score and necrosis ( $n=45, P=0 \cdot 015, H R \quad 0 \cdot 37)$ than with either high 24-gene signature score ( $n=76, H R 0.47$ ) or necrosis ( $n=80, H R 0.43$ ), indicating that combining the two markers identifies the patients that benefit the most from CON. In tumors with either high signature score or necrosis, CON was associated with comparable benefit $(n=111, H R$ 0.49) than in those having either high signature score or necrosis, suggesting that combining the two markers may also identify a wider range of patients that can benefit from hypoxia-targeting therapy.

\section{DISCUSSION}


This study showed that the Lendahl hypoxia signature (15) was predictive of benefit from hypoxic modification but not prognostic in muscle invasive bladder carcinomas. None of the other hypoxia signatures were prognostic or predictive. A de novo 24-gene signature was independently validated in multiple publically available cohorts and the new BCON cohort, and shown to be prognostic and predictive of benefit from the addition of CON to radiotherapy.

Comparison of the de novo 24-gene signature with other published hypoxia signatures showed little overlap. This lack of overlap between genes in hypoxia signatures is well documented and not surprising given the large proportion of the genome that is transcriptionally responsive to changes in oxygenation (3). Four genes (CAV1, P4HA2, DPYSL2 and SLC2A3) from the 24-gene signature also appeared in a hypoxia signature published from another group (14). Similarly, two genes (SLC16A1 and LDLR) were common between the 24-gene signature and a head and neck signature prognostic in multiple cancer types (9). There is one overlap (SLC16A1) between the 24-gene signature and a 26-gene signature derived in HNSCC by our group (13). This 26-gene signature predicted benefit from CON in patients with laryngeal tumors (ARCON phase III trial) but not bladder tumors (BCON) (6). The heterogeneity between signatures could reflect the importance of different biological pathways involved in the tumor hypoxia response in different cancer types. There is likely to be tissue-dependent patterns of transcription modifiers interacting with HIF, as shown recently for the effects of the BET inhibitor JQ1, which modifies a subset of HIF target genes (22).

The limited overlap could also result from differences in the methods for signature derivation (e.g. different starting seeds), and the use of cell lines versus clinical samples 
from different tumor types. On the other hand, any commonality observed is likely due to the conserved portion of the transcriptional response to hypoxia which is independent of hypoxia output $(7,14,15,23)$. Harris et al. (3) performed a systematic review and identified 32 published hypoxia gene signatures. No gene was found in all signatures, but 20 were identified as being the most prevalent. One of these 20 genes, P4HA2, was in the 24-gene signature.

Hypoxia can promote the stabilization of genes that promote somatic copy number gains in tumors. It has been suggested that generation of transient copy number gains could be an adaptive cellular response of cells to stresses such as hypoxia and therefore providing a mechanism for the generation of tumor heterogeneity (24). Our work showed no association between the expression of the 24 genes in our signature with copy number variation in the 24 genes showing no direct association, i.e. the genes do not appear to be modified at the DNA level. In contrast, there appeared to be direct associations with methylation status for the 24-genes with higher methylation associated with lower expression of the individual genes, consistent with a gene silencing effect. Hypoxia leads to epigenetic alterations and some genes are transcriptionally repressed under hypoxia due to histone modification. Hypoxia, however, has also been linked to a global reduction in methylation (25). Further research would be needed to assess the effects of hypoxia in bladder cancer on global copy number alteration and methylation status.

The 24-gene signature was validated in independent publically available cohorts of 679 patients with fresh frozen tissues. The 24-gene signature has translational relevance as it was validated in FFPE samples, a routine technique for preserving tissues in hospitals. It is widely agreed that degradation and chemical modification of nucleic acids in FFPE samples 
reduce RNA quality (26), which further supports the robustness of the signature in clinical application. The results of the current study clearly indicate that the derived gene signature is worthy of further testing using widely abundant FFPE samples. It is noted that a head and neck hypoxia signature developed by Toustrup et al. (4) was validated in FFPE tumor biopsies, and a signature derived from fresh frozen soft tissue sarcoma samples was also prognostic on FFPE samples (27). Second, our group has already validated a hypoxia signature derived for head and neck cancer using a Taqman Low Density Array approach and shown low intra-assay, inter-assay and intra-tumor variability (13). Third, although the de novo signature was applicable to any hypoxia-targeting therapy, an intervention is available (CON) for use in patients. As our head and neck signature is undergoing prospective qualification in a randomized trial (10), the signature derived here can progress in a similar fashion. The BCON cohort is the first time in the literature that whole tumor transcriptome data have been generated for patients recruited into a trial randomizing patients to radiotherapy alone or with hypoxia modification. This enables proper evaluation and comparison of the predictive performance of the de novo signature together with competing protein markers and clinic-pathological factors. A question of interest in terms of the next steps to take is whether to use the gene signature alone or in combination with another marker. Our analyses showed that a combination of simple histological assessment of necrosis with the 24-gene signature was superior to using either alone. The next step, therefore, would be to carry out a biomarker driven trial using the combination.

The use of median score as cut-off was pre-specified in a power calculation to determine the number of BCON samples for gene expression profiling. In validation of the de novo signature, median score was therefore chosen as threshold for patient stratification to avoid 
bias and to provide balanced groups. The de novo 24-gene signature has reached the statistical significance in terms of predicting the benefit of CON in patients with high signature scores (Table 3, HR 0.47, $95 \% \mathrm{Cl} 0.26-0.86, \mathrm{P}=0.015$ ). A test for interaction between the 24-gene signature and intervention showed significance for the resulting binary hypoxic status (median cut-off, $P=0.0094$ ), confirming that response to intervention is indeed different in patients in high and low hypoxia groups. Therefore, the 24-gene signature with the pre-defined median score cut-off confirms the hypothesis of the study and median score is a clinically relevant cut-off. Other thresholds have been used for hypoxic classification $(4,14,23)$. However, there is no consensus on the ideal method to define tumors as hypoxic and no methodological study to date assessing and comparing the performance of the different methods exists. In a prospective clinical trial the first $\sim 50$ patient samples could be used to generate a median threshold in newer FFPE blocks for classification. A strength of the study is the link with an intervention that could pave the way for a future hypoxia signature driven trial.

In conclusion, a hypoxia gene signature for muscle invasive bladder cancer patients receiving radical radiotherapy was derived. Employed as a potential tool for personalized medicine, the signature identifies more hypoxic tumors that have poorer outcome. The signature also predicts benefit from adding concurrent hypoxic modification to radiotherapy. The signature warrants final qualification in a prospective setting. 


\section{CONTRIBUTORS}

LY carried out the signature generation, bioinformatics analysis, statistical analysis and manuscript preparation. JT contributed to the bioinformatics analysis and manuscript preparation. AE and JJI managed the sample collection and data, carried out RNA extraction and organised the generation of microarray data. HD performed pathological assessments. PJH provided access to the data and contributed to the funding application. JA contributed to the bioinformatics analysis. FMB and ALH contributed to the funding application and interpretation of the data. AC was involved in the supervision of the project. CMLW led the funding application and study design, edited the paper for submission and gave final approval for submission. All authors approved the final version of the manuscript. 


\section{REFERENCE}

1. Kim W-J, Kim S-K, Jeong P, Yun S-J, Cho I-C, Kim IY, et al. A four-gene signature predicts disease progression in muscle invasive bladder cancer. Molecular Medicine. 2011;17:478-85.

2. Choueiri TK, Raghavan D. Chemotherapy for muscle-invasive bladder cancer treated with definitive radiotherapy: persisting uncertainties. Nat Clin Prac Oncol. 2008;5:444-54.

3. Harris BHL, Barberis A, West CML, Buffa FM. Gene expression signatures as biomarkers of tumour hypoxia. Clinical Oncology. 2015;27:547-60.

4. Toustrup K, Sørensen BS, Nordsmark M, Busk M, Wiuf C, Alsner J, et al. Development of a hypoxia gene expression classifier with predictive impact for hypoxic modification of radiotherapy in head and neck cancer. Cancer Research. 2011;71:5923-31.

5. Moeller BJ, Richardson RA, Dewhirst MW. Hypoxia and radiotherapy: opportunities for improved outcomes in cancer treatment. Cancer and Metastasis Reviews. 2007;26:241-8.

6. Eustace A, Mani N, Span PN, Irlam JJ, Taylor J, Betts GNJ, et al. A 26-gene hypoxia signature predicts benefit from hypoxia-modifying therapy in laryngeal cancer but not bladder cancer. Clinical Cancer Research. 2013;19:4879-88.

7. Buffa FM, Harris AL, West CM, Miller CJ. Large meta-analysis of multiple cancers reveals a common, compact and highly prognostic hypoxia metagene. Br J Cancer. 2010;102:428-35.

8. Eustace A, Irlam JJ, Taylor J, Denley H, Agrawal S, Choudhury A, et al. Necrosis predicts benefit from hypoxia-modifying therapy in patients with high risk bladder cancer enrolled in a phase III randomised trial. Radiotherapy and Oncology. 2013;108:40-7.

9. Winter SC, Buffa FM, Silva P, Miller C, Valentine HR, Turley H, et al. Relation of a hypoxia metagene derived from head and neck cancer to prognosis of multiple cancers. Cancer Research. 2007;67:3441-9.

10. Fox NS, Starmans MH, Haider S, Lambin P, Boutros PC. Ensemble analyses improve signatures of tumour hypoxia and reveal inter-platform differences. BMC Bioinformatics. 2014;15:114.

11. Hoskin PJ, Rojas AM, Bentzen SM, Saunders MI. Radiotherapy With Concurrent Carbogen and Nicotinamide in Bladder Carcinoma. Journal of Clinical Oncology. 2010;28:4912-8.

12. The Cancer Genome Atlas Research N, Weinstein JN, Collisson EA, Mills GB, Shaw KRM, Ozenberger BA, et al. The cancer genome atlas pan-cancer analysis project. Nat Genet. 2013;45:1113-20.

13. Betts GNJ, Eustace A, Patiar S, Valentine HR, Irlam J, Ramachandran A, et al. Prospective technical validation and assessment of intra-tumour heterogeneity of a low density array hypoxia gene profile in head and neck squamous cell carcinoma. European Journal of Cancer. 2013;49:15665.

14. Chi J-T, Wang Z, Nuyten DSA, Rodriguez EH, Schaner ME, Salim A, et al. Gene Expression Programs in Response to Hypoxia: Cell Type Specificity and Prognostic Significance in Human Cancers. PLoS Med. 2006;3:e47.

15. Lendahl U, Lee KL, Yang H, Poellinger L. Generating specificity and diversity in the transcriptional response to hypoxia. Nat Rev Genet. 2009;10:821-32.

16. Riester M, Taylor JM, Feifer A, Koppie T, Rosenberg JE, Downey RJ, et al. Combination of a Novel Gene Expression Signature with a Clinical Nomogram Improves the Prediction of Survival in High-Risk Bladder Cancer. Clinical Cancer Research. 2012;18:1323-33.

17. Mitra AP, Lam LL, Ghadessi M, Erho N, Vergara IA, Alshalalfa M, et al. Discovery and validation of novel expression signature for postcystectomy recurrence in high-risk bladder cancer. Journal of the National Cancer Institute. 2014;106.

18. Sanchez-Carbayo M, Socci ND, Lozano J, Saint F, Cordon-Cardo C. Defining Molecular Profiles of Poor Outcome in Patients With Invasive Bladder Cancer Using Oligonucleotide Microarrays. Journal of Clinical Oncology. 2006;24:778-89. 
19. Kim W-J, Kim E-J, Kim S-K, Kim Y-J, Ha Y-S, Jeong P, et al. Predictive value of progressionrelated gene classifier in primary non-muscle invasive bladder cancer. Molecular Cancer. 2010;9:1-9. 20. Masiero M, Simões Filipa C, Han Hee D, Snell C, Peterkin T, Bridges E, et al. A Core Human Primary Tumor Angiogenesis Signature Identifies the Endothelial Orphan Receptor ELTD1 as a Key Regulator of Angiogenesis. Cancer Cell. 2013;24:229-41.

21. Hunter BA, Eustace A, Irlam JJ, Valentine HR, Denley H, Oguejiofor KK, et al. Expression of hypoxia-inducible factor-1[alpha] predicts benefit from hypoxia modification in invasive bladder cancer. Br J Cancer. 2014;111:437-43.

22. da Motta LL, Ledaki I, Purshouse K, Haider S, De Bastiani MA, Baban D, et al. The BET inhibitor JQ1 selectively impairs tumour response to hypoxia and downregulates CA9 and angiogenesis in triple negative breast cancer. Oncogene. 2016.

23. Fardin P, Barla A, Mosci S, Rosasco L, Verri A, Versteeg R, et al. A biology-driven approach identifies the hypoxia gene signature as a predictor of the outcome of neuroblastoma patients. Molecular Cancer. 2010;9:1-15.

24. Mishra S, Whetstine JR. Different Facets of Copy Number Changes: Permanent, Transient, and Adaptive. Molecular and Cellular Biology. 2016;36:1050-63.

25. Ramachandran S, lent J, Göttgens E-L, Krieg A, Hammond E. Epigenetic Therapy for Solid Tumors: Highlighting the Impact of Tumor Hypoxia. Genes. 2015;6:935.

26. Hall JS, Taylor J, Valentine HR, Irlam JJ, Eustace A, Hoskin PJ, et al. Enhanced stability of microRNA expression facilitates classification of FFPE tumour samples exhibiting near total mRNA degradation. Br J Cancer. 2012;107:684-94.

27. Lesluyes T, Pérot G, Largeau MR, Brulard C, Lagarde P, Dapremont V, et al. RNA sequencing validation of the Complexity INdex in SARComas prognostic signature. European Journal of Cancer. 2016;57:104-11.

28. Ragnum HB, Vlatkovic L, Lie AK, Axcrona K, Julin CH, Frikstad KM, et al. The tumour hypoxia marker pimonidazole reflects a transcriptional programme associated with aggressive prostate cancer. Br J Cancer. 2015;112:382-90. 
Table 1 Clinicopathologic details by randomization arm

\begin{tabular}{|c|c|c|c|c|}
\hline Variable & $N$ & $\begin{array}{c}\mathbf{R T} \\
\mathrm{N}=75\end{array}$ & $\begin{array}{c}\text { RT+CON } \\
N=76\end{array}$ & $P$ \\
\hline \multicolumn{5}{|l|}{ Gender } \\
\hline Male & 115 & 55 (73.3\%) & $60(78.9 \%)$ & \\
\hline Female & 36 & $20(26.7 \%)$ & $16(21.1 \%)$ & 0.54 \\
\hline Age (years) & 151 & 75.5 (51.1-87.0) & $75.1(51.5-89.7)$ & 0.66 \\
\hline \multicolumn{5}{|l|}{ T stage } \\
\hline $\mathrm{T} 1$ & 9 & $0(0 \%)$ & 9 (11.8\%) & \\
\hline $\mathrm{T} 2$ & 108 & $54(72.0 \%)$ & $54(71.1 \%)$ & \\
\hline T3 & 30 & $19(25.3 \%)$ & $11(14.5 \%)$ & \\
\hline T4a & 4 & $2(2.7 \%)$ & $2(2.6 \%)$ & 0.01 \\
\hline \multicolumn{5}{|l|}{ Grade } \\
\hline 2 & 1 & 0 (0\%) & $1(1.3 \%)$ & \\
\hline 3 & 150 & 75 (100\%) & 75 (98.7\%) & 0.99 \\
\hline \multicolumn{5}{|l|}{ TURBT } \\
\hline Biopsy & 33 & $15(20.0 \%)$ & $18(23.4 \%)$ & \\
\hline Partial & 58 & $24(32.0 \%)$ & $24(31.6 \%)$ & \\
\hline Complete & 55 & $32(42.7 \%)$ & $33(43.4 \%)$ & \\
\hline No data & 5 & 4 (5.3\%) & 1 (1.3\%) & 0.55 \\
\hline $\mathrm{Hb}(\mathrm{g} / \mathrm{L})$ & 149 & $13.7(9.8-16.9)$ & $13.9(9.5-17.2)$ & 0.42 \\
\hline No data & 2 & $1(1.3 \%)$ & $1(1.3 \%)$ & \\
\hline \multicolumn{5}{|l|}{ Concurrent } \\
\hline \multicolumn{5}{|l|}{ pTis } \\
\hline Absent & 117 & $50(67.7 \%)$ & $67(88.2 \%)$ & \\
\hline Present & 34 & $25(33.3 \%)$ & $9(11.8 \%)$ & 0.003 \\
\hline \multicolumn{5}{|l|}{ Necrosis } \\
\hline Absent & 71 & 39 (52.0\%) & $32(42.1 \%)$ & \\
\hline Present & 80 & $36(48.0 \%)$ & 44 (57.9\%) & 0.29 \\
\hline \multicolumn{5}{|c|}{$\begin{array}{l}\text { Data represent median (range) or } n(\%) \\
\text { Abbreviations: } \mathrm{RT}=\text { radiotherapy, CON= carbogen and nicotinamide, TURBT= transurethral } \\
\text { resection of bladder tumor, } \mathrm{Hb}=\text { hemoglobin, } \mathrm{pTis}=\text { carcinoma in situ } \\
\text { Most patients ( } 151 ; 99 \%) \text { received } \geq 90 \% \text { of the prescribed RT. In the experimental arm, } 66 \\
(86 \%) \text { patients received } \geq 90 \% \text { of the stipulated carbogen doses and } 50(65 \%) \text { patients } \\
\text { received } \geq 90 \% \text { of the stipulated nicotinamide doses. All analyses were conducted on an } \\
\text { "intention to treat" basis. }\end{array}$} \\
\hline
\end{tabular}


Table 2 Prognostic and predictive significance of the literature signatures for LPFS analysis of BCON cohort

\begin{tabular}{|c|c|c|}
\hline Signature & BCON Prognosis $^{a}$ & $\begin{array}{l}\text { BCON (high-hypoxia patients) } \\
\text { Prediction of Benefit }\end{array}$ \\
\hline Ragnum et al. (28) & 0.882 & 0.389 \\
\hline Buffa et al. (7) & 0.907 & 0.827 \\
\hline Winter et al. (9) & 0.438 & 0.434 \\
\hline Betts et al. (13) & 0.569 & 0.330 \\
\hline Toustrup et al. (4) & 0.252 & 0.856 \\
\hline Toustrup et al. ${ }^{b}(4)$ & 0.653 & 0.664 \\
\hline Chi et al. (14) & 0.120 & 0.078 \\
\hline Lendahl et al. (15) & 0.093 . & $0.038 *$ \\
\hline Mitra et al. (17) & 0.960 & 0.530 \\
\hline Riester et al. (16) & $0.002 * *$ & $0.032 *$ \\
\hline Sanchez-Carbayo et al. (18) & 0.260 & 0.140 \\
\hline Kim et al. (19) & 0.478 & 0.541 \\
\hline Kim et al. (1) & 0.855 & 0.880 \\
\hline
\end{tabular}

Significance codes: 0.001 "**” 0.01 "*” 0.05 "." 0.1

${ }^{a}$ Prognosis was evaluated in patients receiving radiotherapy only, while prediction of benefit of CON was assessed with both high-hypoxia and low-hypoxia groups.

${ }^{\mathrm{b}}$ Classification of samples into more or less hypoxic using the centroid method described in Toustrup et al. 
Table 3 Multivariate analysis of hypoxia signature and pathological variables for BCON study

\begin{tabular}{|c|c|c|c|c|c|}
\hline & \multirow[t]{2}{*}{ Variable } & \multicolumn{2}{|c|}{ Univariate } & \multicolumn{2}{|c|}{ Multivariate } \\
\hline & & $\begin{array}{c}\text { HR } \\
(95 \% \mathrm{Cl})\end{array}$ & $P$ value & $\begin{array}{c}\mathrm{HR} \\
(95 \% \mathrm{Cl})\end{array}$ & $P$ value \\
\hline \multirow{6}{*}{ RT arm } & Hypoxia & $\begin{array}{c}2.37 \\
(1.26-4.47)\end{array}$ & $0.0076^{* *}$ & $\begin{array}{c}2.25 \\
(1.16-4.39)\end{array}$ & $0.017^{*}$ \\
\hline & Stage 3 or $4 a$ & $\begin{array}{c}0.59 \\
(0.28-1.23)\end{array}$ & 0.18 & $\begin{array}{c}0.44 \\
(0.21-0.95)\end{array}$ & $0.036^{*}$ \\
\hline & Male & $\begin{array}{c}0.86 \\
(0.45-1.64)\end{array}$ & 0.65 & $\begin{array}{c}0.82 \\
(0.42-1.63)\end{array}$ & 0.58 \\
\hline & Age & $\begin{array}{c}1.02 \\
(0.98-1.06)\end{array}$ & 0.34 & $\begin{array}{c}1.02 \\
(0.98-1.06)\end{array}$ & 0.40 \\
\hline & $\mathrm{CIS}$ & $\begin{array}{c}2.06 \\
(1.13-3.74)\end{array}$ & $0.018^{*}$ & $\begin{array}{c}2.23 \\
(1.18-4.22)\end{array}$ & $0.013^{*}$ \\
\hline & Necrosis & $\begin{array}{c}2.10 \\
(1.16-3.80)\end{array}$ & $0.015^{*}$ & $\begin{array}{c}2.21 \\
(1.18-4.16)\end{array}$ & $0.013^{*}$ \\
\hline \multirow{6}{*}{ High-hypoxia } & CON & $\begin{array}{c}0.47 \\
(0.26-0.86)\end{array}$ & $0.015^{*}$ & $\begin{array}{c}0.52 \\
(0.26-1.02)\end{array}$ & 0.058 \\
\hline & Stage 3 or $4 a$ & $\begin{array}{c}0.58 \\
(0.29-1.17)\end{array}$ & 0.13 & $\begin{array}{c}0.49 \\
(0.24-1.01)\end{array}$ & 0.052 \\
\hline & Male & $\begin{array}{c}0.90 \\
(0.49-1.67)\end{array}$ & 0.75 & $\begin{array}{c}0.93 \\
(0.49-1.74)\end{array}$ & 0.81 \\
\hline & Age & $\begin{array}{c}1.03 \\
(0.99-1.08)\end{array}$ & 0.094 & $\begin{array}{c}1.03 \\
(0.99-1.08)\end{array}$ & 0.14 \\
\hline & $\mathrm{CIS}$ & $\begin{array}{c}2.93 \\
(1.59-5.39)\end{array}$ & $0.0006 * * *$ & $\begin{array}{c}2.63 \\
(1.30-5.32)\end{array}$ & $0.0069 * *$ \\
\hline & Necrosis & $\begin{array}{c}1.17 \\
(0.66-2.1)\end{array}$ & 0.59 & $\begin{array}{c}1.47 \\
(0.78-2.77)\end{array}$ & 0.24 \\
\hline
\end{tabular}

Significance codes: 0.0001 “***” 0.001 “**” 0.01 “*” 0.05 “.” 0.1 


\section{Titles and legends to figures}

Figure 1. Kaplan-Meier plots for independent validations of the 24-gene hypoxia signature in chemotherapy or surgery-treated cohorts.

Figure 2. Kaplan-Meier plots for BCON patients receiving A: RT alone; B: RT plus CON. Patients were stratified into high-hypoxia and low-hypoxia by the 24-gene signature. Patients with persistent muscle-invasive disease or with no cystoscopy post treatment had their time set to zero.

Figure 3. Kaplan-Meier plots for BCON patients stratified as either A: high-hypoxia; or B: low-hypoxia by the 24-gene signature. In each group, patients received either RT or RT plus CON. Patients with persistent muscle-invasive disease or with no cystoscopy post treatment had their time set to zero. 


\section{Figure 1}

\section{A: GSE5287}

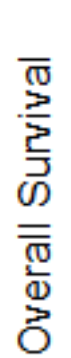

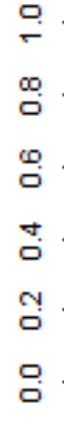
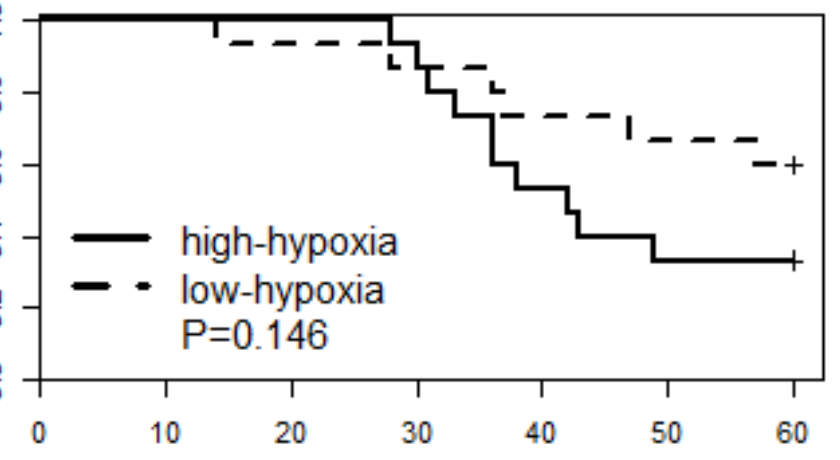

Time in Months

\# at risk high low 15$$
\begin{aligned}
& 14 \\
& 13
\end{aligned}
$$

$$
\begin{aligned}
& 15 \\
& 15
\end{aligned}
$$$$
\begin{aligned}
& 15 \\
& 14
\end{aligned}
$$$$
\begin{gathered}
8 \\
11
\end{gathered}
$$

\section{C: GSE31684}
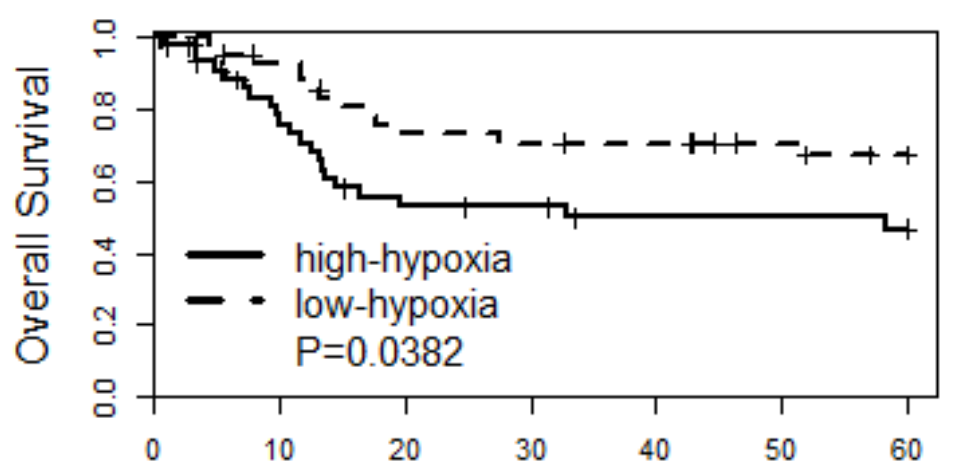

Time in Months

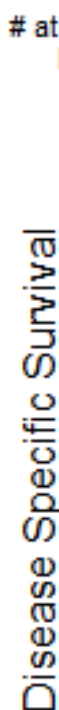

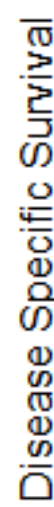$$
\text { ○ }
$$

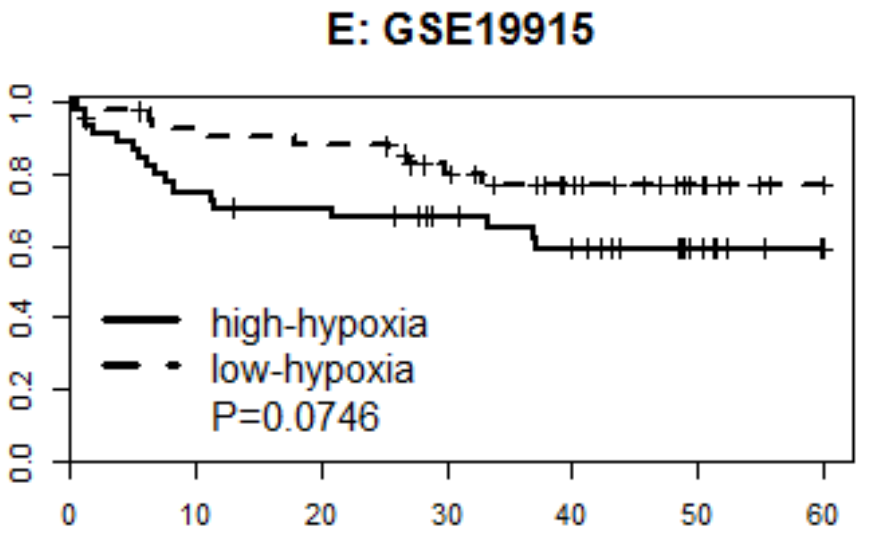

\# at risk high low 42
Time in Months

$\begin{array}{lllllll}47 & 30 & 20 & 19 & 16 & 16 & 15 \\ 46 & 38 & 29 & 28 & 27 & 23 & 20\end{array}$

\section{E: GSE19915}

$\begin{array}{lll}30 & 25 & 19\end{array}$

$\begin{array}{ll}30 & 25 \\ 36 & 29\end{array}$

19
20

$\begin{array}{cc}5 & 5 \\ 10 & 9\end{array}$

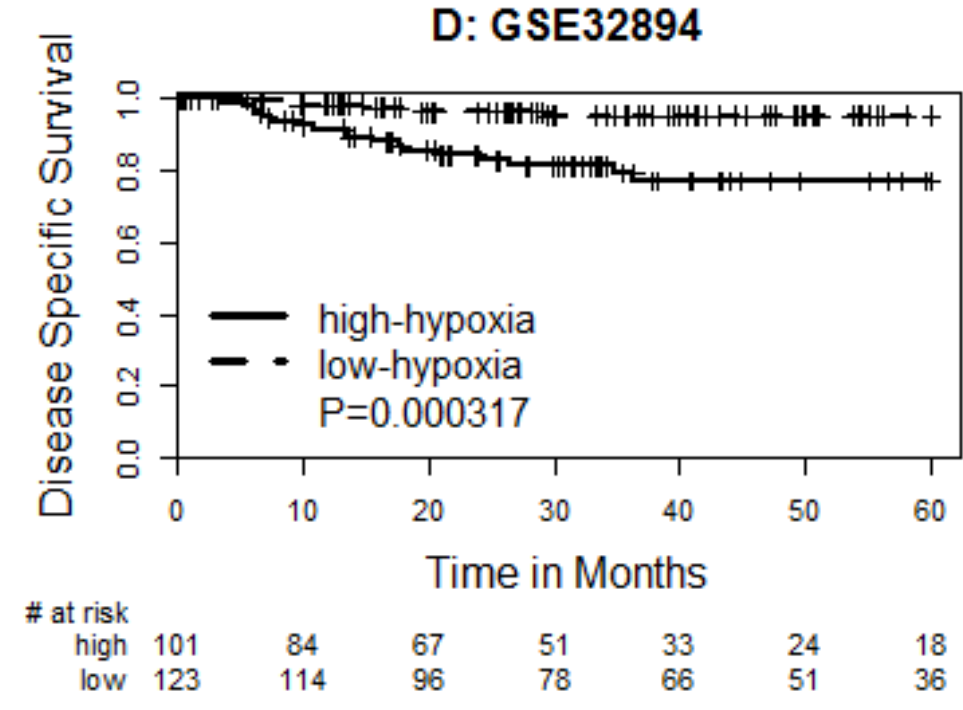

\section{F: GSE1827}

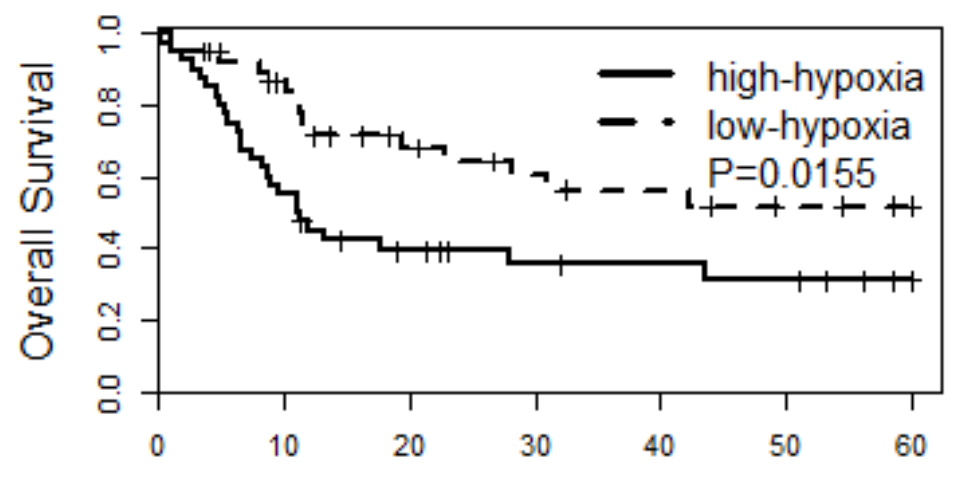

Time in Months

$\begin{array}{rllccccc}\text { \# at risk } & & & & & & \\ \text { high } & 40 & 22 & 13 & 9 & 8 & 7 & 3 \\ \text { low } & 40 & 29 & 19 & 15 & 13 & 10 & 8\end{array}$




\section{Figure 2}

\section{A: BCON RT Arm}

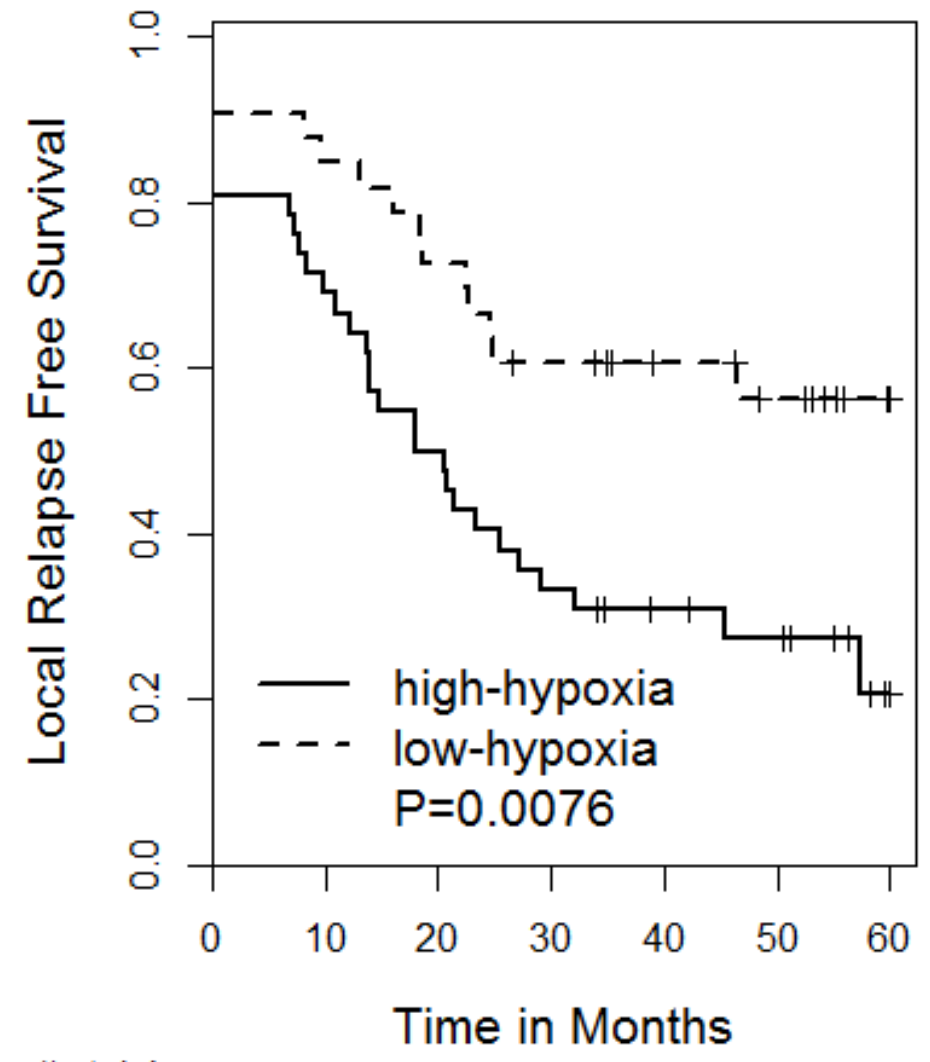

\# at risk

$\begin{array}{lllllll}\text { high } 42 & 29 & 21 & 14 & 10 & 8 & 1\end{array}$

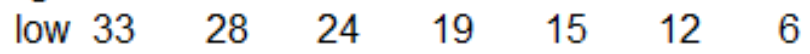

B: BCON CON Arm

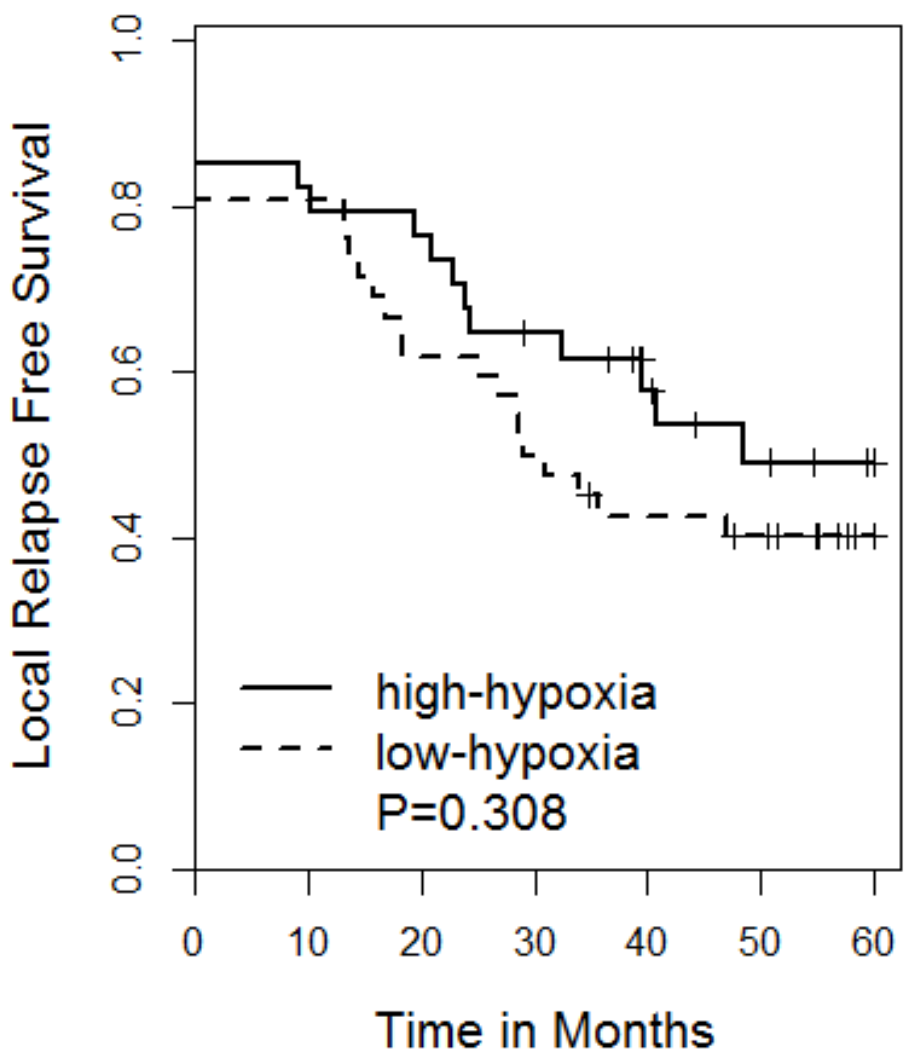

\# at risk

$\begin{array}{lllllll}\text { high } 34 & 28 & 26 & 21 & 15 & 11 & 7\end{array}$ low $42 \quad 34 \quad 26 \quad 21 \quad 17 \quad 15 \quad 8$ 


\section{Figure 3}

\section{A: BCON High Hypoxia Patients}

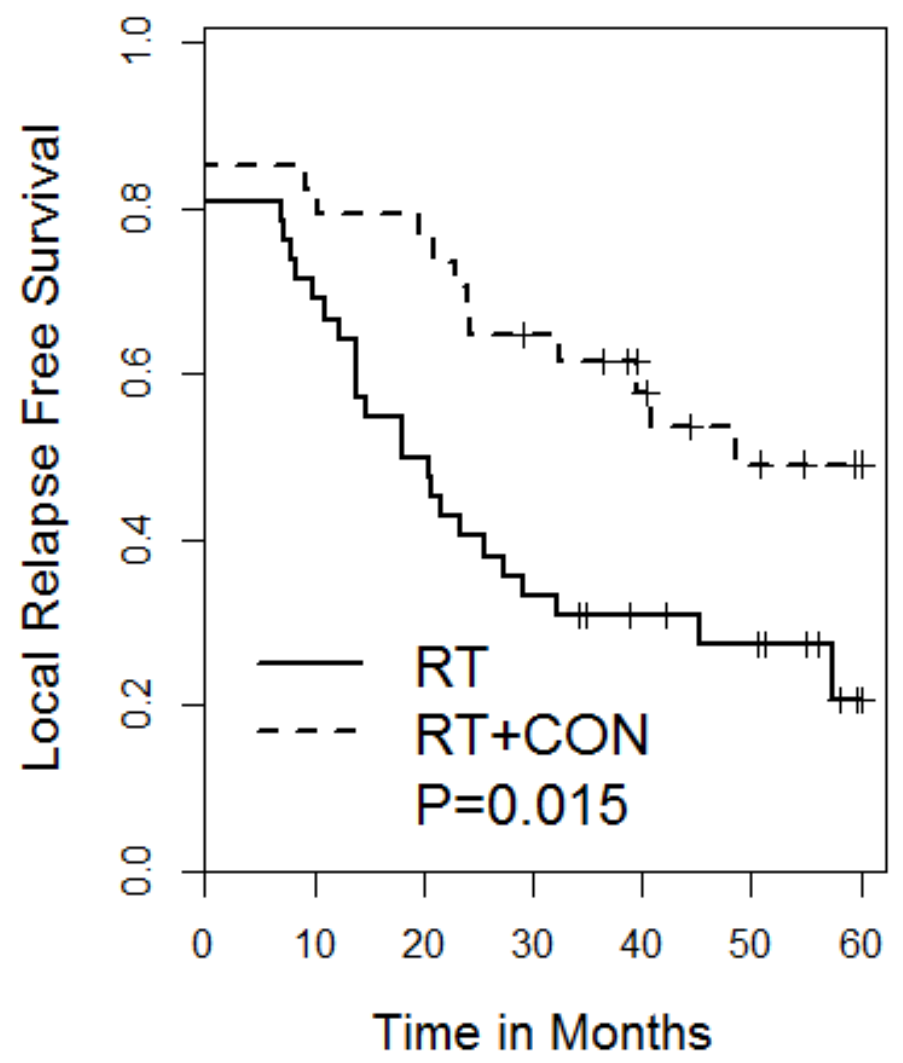

\# at risk

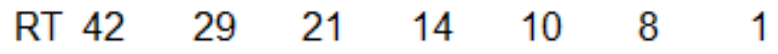

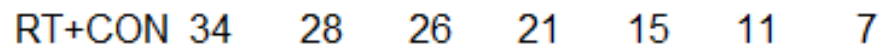

B: BCON Low Hypoxia Patients

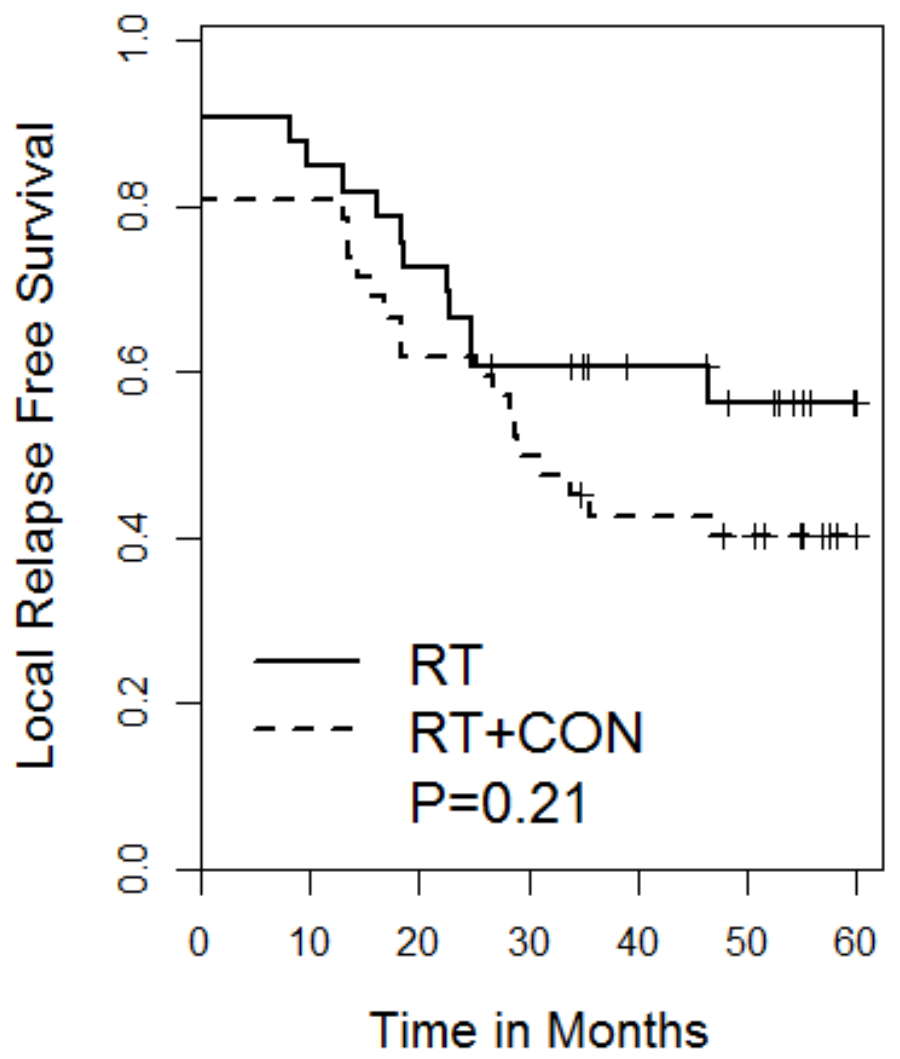

\# at risk

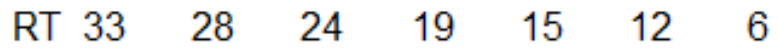
$\begin{array}{llllllll}\mathrm{RT}+\mathrm{CON} & 42 & 34 & 26 & 21 & 17 & 15 & 8\end{array}$ 


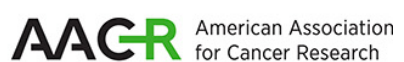

\section{Clinical Cancer Research}

\section{A Gene Signature for Selecting Benefit from Hypoxia Modification of Radiotherapy for High Risk Bladder Cancer Patients}

Lingjian Yang, Janet Taylor, Amanda Eustace, et al.

Clin Cancer Res Published OnlineFirst April 11, 2017.

Updated version Access the most recent version of this article at: doi:10.1158/1078-0432.CCR-17-0038

Supplementary Access the most recent supplemental material at:

Material http://clincancerres.aacrjournals.org/content/suppl/2017/04/11/1078-0432.CCR-17-0038.DC1

Author Author manuscripts have been peer reviewed and accepted for publication but have not yet been Manuscript edited.

E-mail alerts Sign up to receive free email-alerts related to this article or journal.

Reprints and To order reprints of this article or to subscribe to the journal, contact the AACR Publications Subscriptions Department at pubs@aacr.org.

Permissions To request permission to re-use all or part of this article, contact the AACR Publications Department at permissions@aacr.org. 